\title{
Further Generalized Beta Function with Three Parameters Mittag- Leffler Function
}

\section{Salem Saleh Barahmah}

Department of Mathematics, Aden University, Aden, Yemen; e-mail: salemalqasemi@yahoo.com

\begin{abstract}
The purpose of the present paper is to introduce a new extension of extended Beta function by product of two Mittag-Leffler functions. Further, we present certain results including summation formulas, integral representations and Mellin transform.
\end{abstract}

\section{Introduction}

The classical Beta function is defined in [11]:

$$
\begin{aligned}
B(x, y) & =\int_{0}^{1} t^{x-1}(1-t)^{y-1} d t, \quad(\operatorname{Re}(x)>0, \operatorname{Re}(y)>0) \\
& =\frac{\Gamma(x) \Gamma(y)}{\Gamma(x+y)}, \quad \operatorname{Re}(x)>0, \operatorname{Re}(y)>0
\end{aligned}
$$

where $\Gamma(x)$ denotes the classical Gamma function defined in [11]

$$
\Gamma(x)=\int_{0}^{\infty} t^{x-1} e^{-t} d t, \quad(\operatorname{Re}(x)>0) .
$$

In 1903, Mittag-Leffler [6] introduced the function $E_{\alpha}(z)$ defined by

Received: November 19, 2018; Accepted: December 3, 2018

2010 Mathematics Subject Classification: 33B20, 33C20, 33B15, 33C05.

Keywords and phrases: extended Beta function, Mittag-Leffler function, summation formulas, integral representations, Mellin transform.

Copyright (c) 2019 Salem Saleh Barahmah. This is an open access article distributed under the Creative Commons Attribution License, which permits unrestricted use, distribution, and reproduction in any medium, provided the original work is properly cited. 


$$
\begin{gathered}
E_{\alpha}(z)=\sum_{n=0}^{\infty} \frac{z^{n}}{\Gamma(\alpha n+1)}, \quad \alpha>0, z \in \mathbb{C} . \\
E_{1}(z)=e^{z} .
\end{gathered}
$$

The generalized Mittag-Leffler function $E_{\alpha, \beta}(z)[12]$ is defined by

$$
\begin{gathered}
E_{\alpha, \beta}(z)=\sum_{n=0}^{\infty} \frac{z^{n}}{\Gamma(\alpha n+\beta)}, \quad(\alpha, \beta \in \mathbb{C}, \operatorname{Re}(\alpha)>0, \operatorname{Re}(\beta)>0) . \\
E_{\alpha, 1}(z)=E_{\alpha}(z) .
\end{gathered}
$$

Afterward, Prabhakar [7] defined the generalized Mittag-Leffler function by

$$
\begin{gathered}
E_{\alpha, \beta}^{\gamma}(z)=\sum_{n=0}^{\infty} \frac{(\gamma)_{n}}{\Gamma(\alpha n+\beta)} \frac{z^{n}}{n !}, \quad(\alpha, \beta, \gamma \in \mathbb{C}, \operatorname{Re}(\alpha)>0) . \\
E_{\alpha, \beta}^{1}(z)=E_{\alpha, \beta}(z) .
\end{gathered}
$$

Recently, many authors have introduced certain extensions of extended Gamma and Beta functions (1.3) and (1.1) (see [1, 2, 3, 4, 5, 8, 9, 10]).

The following two extended Beta functions are introduced by Chaudhry et al. [4] and Choi et al. [5] respectively:

$$
\begin{gathered}
B(x, y ; p)=\int_{0}^{1} t^{x-1}(1-t)^{y-1} \exp \left(\frac{-p}{t(1-t)}\right) d t, \\
(\operatorname{Re}(p)>0, \operatorname{Re}(x)>0, \operatorname{Re}(y)>0) .
\end{gathered}
$$

and

$$
\begin{gathered}
B(x, y ; p ; q)=\int_{0}^{1} t^{x-1}(1-t)^{y-1} \exp \left(\frac{-p}{t}-\frac{q}{(1-t)}\right) d t, \\
(\operatorname{Re}(p)>0, \operatorname{Re}(q)>0, \operatorname{Re}(x)>0, \operatorname{Re}(y)>0) .
\end{gathered}
$$

The following extended Beta function is given by Rahman et al. [9]:

$$
B_{p, q}^{\alpha}(x, y)=\int_{0}^{1} t^{x-1}(1-t)^{y-1} E_{\alpha}\left(-\frac{p}{t}\right) E_{\alpha}\left(-\frac{q}{(1-t)}\right) d t
$$




$$
(\operatorname{Re}(x)>0, \operatorname{Re}(y)>0, \alpha>0, p, q \geq 0) .
$$

Also, Al-Gonah and Mohammed [1] introduced the following extension of Gamma and Beta functions:

$$
\begin{gathered}
B_{p}^{(\alpha, \beta, \gamma)}(x, y)=\int_{0}^{1} t^{x-1}(1-t)^{y-1} E_{\alpha, \beta}^{\gamma}\left(-\frac{p}{t(1-t)}\right) d t \\
\operatorname{Re}(x)>0, \operatorname{Re}(y)>0, \operatorname{Re}(\alpha)>0, \operatorname{Re}(p) \geq 0
\end{gathered}
$$

and

$$
\begin{gathered}
\Gamma_{p}^{(\alpha, \beta, \gamma)}(x)=\int_{0}^{\infty} t^{x-1} E_{\alpha, \beta}^{\gamma}\left(-t-\frac{p}{t}\right) d t, \quad(\operatorname{Re}(p)>0) \\
(\operatorname{Re}(x)>0, \operatorname{Re}(\alpha)>0, \operatorname{Re}(p)>0) .
\end{gathered}
$$

Afterwards, Atash et al. [2] introduced the following extension of Beta function:

$$
\begin{gathered}
B_{p, q}^{(\alpha, \beta)}(x, y)=\int_{0}^{t} t^{x-1}(1-t)^{y-1} E_{\alpha, \beta}\left(-\frac{p}{t}\right) E_{\alpha, \beta}\left(-\frac{q}{(1-t)}\right) d t, \\
(\operatorname{Re}(x)>0, \operatorname{Re}(y)>0, \operatorname{Re}(\alpha)>0, \beta \in \mathbb{C}, p, q \geq 0) .
\end{gathered}
$$
form:

In the present paper, we introduce a new extension of Beta functions in the following

$$
\begin{gathered}
B_{p, q}^{(\gamma ; \alpha, \beta)}(x, y)=\int_{0}^{1} t^{x-1}(1-t)^{y-1} E_{\alpha, \beta}^{\gamma}\left(-\frac{p}{t}\right) E_{\alpha, \beta}^{\gamma}\left(-\frac{q}{(1-t)}\right) d t, \\
(\operatorname{Re}(x)>0, \operatorname{Re}(y)>0, \operatorname{Re}(\alpha)>0, p, q \geq 0) .
\end{gathered}
$$

\section{Remark 1.}

(i) For $\gamma=1$, equation (1.13) reduces to extended Gamma function (1.12).

(ii) For $\gamma=\beta=1$, equation (1.13) reduces to extended Beta function (1.9).

(iii) For $\gamma=\alpha=\beta=1$, equation (1.13) reduces to extended Beta function (1.8).

(iv) For $\gamma=\alpha=\beta=1$ and $p=q$, equation (1.13) reduces to extended Beta function (1.7).

(v) For $\gamma=\alpha=\beta=1$ and $p=q=0$, equation (1.13) reduces to the classical Beta function (1.1). 


\section{Properties and Formulas}

In this section, we present certain properties of extension Beta function including summation formulas, integral representations and Mellin transform.

Theorem 1. The following summation formulas hold true:

(i) $B_{p, q}^{(\gamma ; \alpha, \beta)}(x, 1-y)=\sum_{n=0}^{\infty} \frac{(y)_{n}}{n !} B_{p, q}^{(\gamma ; \alpha, \beta)}(x+n, 1)$,

(ii) $B_{p, q}^{(\gamma ; \alpha, \beta)}(x, y)=\sum_{n=0}^{\infty} B_{p, q}^{(\gamma ; \alpha, \beta)}(x+n, y+1)$.

Proof. From (1.13), we have

$$
B_{p, q}^{(\gamma ; \alpha, \beta)}(x, y-1)=\int_{0}^{1} t^{x-1}(1-t)^{-y} E_{\alpha, \beta}^{\gamma}\left(-\frac{p}{t}\right) E_{\alpha, \beta}^{\gamma}\left(-\frac{q}{(1-t)}\right) d t
$$

Using the generalized binomial theorem

$$
(1-t)^{-y}=\sum_{n=0}^{\infty} \frac{(y)_{n}}{n !} t^{n}, \quad|t|<1
$$

we obtain

$$
B_{p, q}^{(\gamma ; \alpha, \beta)}(x, 1-y)=\int_{0}^{1} \sum_{n=0}^{\infty} \frac{(y)_{n}}{n !} t^{x+n-1} E_{\alpha, \beta}^{\gamma}\left(-\frac{p}{t}\right) E_{\alpha, \beta}^{\gamma}\left(-\frac{q}{(1-t)}\right) d t
$$

Now, interchanging the order of summation and integration in the above equation and using (1.13), we obtain the required result (2.1). The proof of (2.2) is similar to that of (2.1) and we use here the generalized binomial theorem

$$
(1-t)^{y-1}=(1-t)^{y} \sum_{n=0}^{\infty} t^{n}, \quad|t|<1 .
$$

Theorem 2. The following summation formula holds true:

$$
\sum_{k=0}^{n}\left(\begin{array}{l}
n \\
k
\end{array}\right) B_{p, q}^{(\gamma ; \alpha, \beta)}(x+k, y+n-k)=B_{p, q}^{(\gamma ; \alpha, \beta)}(x, y), \quad n \in \mathbb{N}_{0} .
$$


Proof. For proof of (2.3) we use the mathematical induction on $\left(n \in \mathbb{N}_{0}\right)$ as follows:

Clearly, for $n=0$ the equation (2.3) holds.

For $n=1$, we have

$$
\begin{aligned}
& B_{p, q}^{(\gamma ; \alpha, \beta)}(x+1, y)+B_{p, q}^{(\gamma ; \alpha, \beta)}(x, y+1) \\
= & \int_{0}^{1}\left\{t^{x}(1-t)^{y-1}+t^{x-1}(1-t)^{y}\right\} E_{\alpha, \beta}^{\gamma}\left(-\frac{p}{t}\right) E_{\alpha, \beta}^{\gamma}\left(-\frac{q}{(1-t)}\right) d t \\
= & \int_{0}^{1} t^{x-1}(1-t)^{y-1}\{t+(1-t)\} E_{\alpha, \beta}^{\gamma}\left(-\frac{p}{t}\right) E_{\alpha, \beta}^{\gamma}\left(-\frac{q}{(1-t)}\right) d t \\
= & \int_{0}^{1} t^{x-1}(1-t)^{y-1} E_{\alpha, \beta}^{\gamma}\left(-\frac{p}{t}\right) E_{\alpha, \beta}^{\gamma}\left(-\frac{q}{(1-t)}\right) d t \\
= & B_{p, q}^{(\gamma ; \alpha, \beta)}(x, y) .
\end{aligned}
$$

Therefore, the equation (2.3) holds for $n=1$.

Continuing this process for all $\left(n \in \mathbb{N}_{0}\right)$, we finally obtain the desired relation (2.3).

\section{Remark 2.}

(i) If $\gamma=1$, then (2.4) reduces to a known result of Atash et al. [2].

(ii) If $\gamma=\alpha=\beta=1$ and $p=q$, then (2.4) reduces to a known result of Chaudhry et al. [4, p.23 (3.1)].

(iii) If $\gamma=\alpha=\beta=1$, then (2.4) reduces to a known result of Choi et al. [5, p.362 (3.1)].

(iv) If $\gamma=\beta=1$, then (2.4) reduces to a known result of Rahman et al. [9, p.7 (3.1)].

(v) If $\gamma=\alpha=\beta=1$ and $p=q=0$, then (2.4) reduces to a known result for the classical Beta function.

Theorem 3. The following integral formula holds true:

$$
\Gamma_{0}^{(\alpha, \beta, \gamma)}(u) \Gamma_{0}^{(\alpha, \beta, \gamma)}(v)=\frac{1}{B(x+u, y+v)} \int_{0}^{\infty} \int_{0}^{\infty} p^{u-1} q^{v-1} B_{p, q}^{(\gamma ; \alpha, \beta)}(x, y) d p d q
$$




$$
\begin{aligned}
(\operatorname{Re}(x+u) & >0, \operatorname{Re}(y+v)>0, \operatorname{Re}(\alpha)>0, \\
& \operatorname{Re}(\beta)>0, \operatorname{Re}(p), \operatorname{Re}(q)>0, \operatorname{Re}(u), \operatorname{Re}(v)>0) .
\end{aligned}
$$

Proof. From (1.11), we have

$$
\begin{aligned}
\Gamma_{0}^{(\alpha, \beta, \gamma)}(u) \Gamma_{0}^{(\alpha, \beta, \gamma)}(v)= & \frac{B(x+u, y+v)}{B(x+u, y+v)} \int_{0}^{\infty} \int_{0}^{\infty} w^{u-1} z^{v-1} E_{\alpha, \beta}^{\gamma}(-w) E_{\alpha, \beta}^{\gamma}(-z) d w d z \\
= & \frac{1}{B(x+u, y+v)} \int_{0}^{1} t^{x+u-1}(1-t)^{y+v-1} \\
& \times\left\{\int_{0}^{\infty} \int_{0}^{\infty} w^{u-1} z^{v-1} E_{\alpha, \beta}^{\gamma}(-w) E_{\alpha, \beta}^{\gamma}(-z) d w d z\right\} d t .
\end{aligned}
$$

Substituting $w=\frac{p}{t}, z=\frac{q}{(1-t)}$, we get

$$
\begin{aligned}
\Gamma_{0}^{(\alpha, \beta, \gamma)}(u) \Gamma_{0}^{(\alpha, \beta, \gamma)}(v)= & \frac{1}{B(x+u, y+v)} \int_{0}^{1} t^{x-1}(1-t)^{y-1} \\
& \times\left\{\int_{0}^{\infty} \int_{0}^{\infty} p^{u-1} q^{\nu-1} E_{\alpha, \beta}^{\gamma}\left(-\frac{p}{t}\right) E_{\alpha, \beta}^{\gamma}\left(-\frac{q}{(1-t)}\right) d p d q\right\} d t,
\end{aligned}
$$

Under stated conditions of the above integral, the order of integration can be interchanging. Therefore, we have

$$
\begin{aligned}
\Gamma_{0}^{(\alpha, \beta, \gamma)}(u) \Gamma_{0}^{(\alpha, \beta, \gamma)}(v)= & \frac{1}{B(x+u, y+v)} \int_{0}^{\infty} \int_{0}^{\infty} p^{u-1} q^{\nu-1} \\
& \times\left\{\int_{0}^{1} t^{x-1}(1-t)^{y-1} E_{\alpha, \beta}^{\gamma}\left(-\frac{p}{t}\right) E_{\alpha, \beta}^{\gamma}\left(-\frac{q}{(1-t)}\right) d t\right\} d p d q .
\end{aligned}
$$

Finally, using the result (1.13), we obtain the desired result.

\section{Remark 3.}

(i) The special case $\gamma=1$ of (2.5) reduces to a known result of Atash et al. [2].

(ii) The special case $\gamma=\alpha=\beta=1$ of (2.5) reduces to a known result of Choi et al. [5, p.360 (2.1)]. 
Theorem 4. The following integral representations for $B_{p, q}^{(\gamma ; \alpha, \beta)}(x, y)$ holds true:

(i) $B_{p, q}^{(\gamma ; \alpha, \beta)}(x, y)=2 \int_{0}^{\frac{\pi}{2}} \cos ^{2 x-1} \theta \sin ^{2 y-1} \theta E_{\alpha, \beta}^{\gamma}\left(-\frac{p}{\cos ^{2} \theta}\right) E_{\alpha, \beta}^{\gamma}\left(-\frac{q}{\sin ^{2} \theta}\right) d \theta$,

(ii) $B_{p, q}^{(\gamma ; \alpha, \beta)}(x, y)=\int_{0}^{\infty} \frac{u^{x-1}}{(1+u)^{x+y}} E_{\alpha, \beta}^{\gamma}\left(-\frac{p(1+u)}{u}\right) E_{\alpha, \beta}^{\gamma}(-q(1+u)) d u$,

(iii) $B_{p, q}^{(\gamma ; \alpha, \beta)}(x, y)=(c-a)^{1-x-y} \int_{a}^{c}(u-a)^{x-1}(c-u)^{x-1}$

$$
\times E_{\alpha, \beta}^{\gamma}\left(-\frac{p(c-a)}{(u-a)}\right) E_{\alpha, \beta}^{\gamma}\left(-\frac{q(c-a)}{(c-u)}\right) d u .
$$

Proof. To prove the formula (2.6), putting $t=\cos ^{2} \theta$ in (1.13), we have

$$
\begin{aligned}
B_{p, q}^{(\gamma ; \alpha, \beta)}(x, y)= & 2 \int_{0}^{\frac{\pi}{2}} \cos ^{2 x-2} \theta \sin ^{2 y-2} \theta E_{\alpha, \beta}^{\gamma}\left(\frac{p}{\cos ^{2} \theta}\right) \\
& \times E_{\alpha, \beta}^{\gamma}\left(-\frac{q}{1-\cos ^{2} \theta}\right) \cos \theta \sin \theta d \theta, \\
B_{p, q}^{(\gamma ; \alpha, \beta)}(x, y)= & 2 \int_{0}^{\frac{\pi}{2}} \cos ^{2 x-1} \theta \sin ^{2 y-1} \theta E_{\alpha, \beta}^{\gamma}\left(-\frac{p}{\cos ^{2} \theta}\right) E_{\alpha, \beta}^{\gamma}\left(-\frac{q}{\sin ^{2} \theta}\right) d \theta .
\end{aligned}
$$

Similarly, the formulas (2.7) and (2.8) can be proved by taking the transformation $t=\frac{u}{1+u}$ and $t=\frac{u-a}{c-a}$ in (1.13), respectively.

Theorem 5. The following Mellin transformation formula holds true:

$$
\begin{gathered}
B_{p, q}^{(\gamma ; \alpha, \beta)}(x, y)=\frac{1}{(2 \pi i)^{2}} \int_{\gamma_{1}-i \infty}^{\gamma_{1}+i \infty} \int_{\gamma_{2}-i \infty}^{\gamma_{2}+i \infty} B(x+u, y+v) \Gamma_{0}^{(\alpha, \beta, \gamma)}(u) \Gamma_{0}^{(\alpha, \beta, \gamma)}(v) \\
\quad \times p^{-r} q^{-s} d u d v \\
\left(\operatorname{Re}(x)>0, \operatorname{Re}(y)>0, \operatorname{Re}(\alpha)>0, \operatorname{Re}(\beta)>0, p, q \geq 0, \gamma_{1}, \gamma_{2}>0\right)
\end{gathered}
$$


Proof. Clearly, by applying the definition of Mellin transformation to the right hand side of (2.5), we obtain

$$
\begin{gathered}
\mathcal{M}\left\{B_{p, q}^{(\gamma ; \alpha, \beta)}(x, y) ; p \rightarrow u, q \rightarrow v\right\} \\
=B(x+u, y+v) \Gamma_{0}^{(\alpha, \beta, \gamma)}(u) \Gamma_{0}^{(\alpha, \beta, \gamma)}(v), \\
(\operatorname{Re}(x+u)>0, \operatorname{Re}(y+v)>0, \operatorname{Re}(\alpha)>0, \operatorname{Re}(\beta)>0, \\
\operatorname{Re}(p), \operatorname{Re}(q)>0, \operatorname{Re}(u), \operatorname{Re}(v)>0) .
\end{gathered}
$$

Now, by taking the inverse Mellin transformation on both sides of (2.10), we get the desired result.

\section{Remark 4.}

(i) If $\gamma=1$, then (2.10) reduces to a known result of Atash et al. [2].

(ii) If $\gamma=\beta=1$, then (2.10) reduces to a known result of Rahman et al. [9, p.5 (2.2)].

(iii) If $\gamma=\alpha=\beta=1$ and $p=q$, then (2.11) reduces to a known result of Chaudhry et al. [4, p.28 (5.1)].

(iv) If $\gamma=\alpha=\beta=1$, then (2.11) reduces to a known result of Choi et al. [5, p.365 (4.2)].

\section{References}

[1] A. A. Al-Gonah and W. K. Mohammed, A new extension of extended Gamma and Beta functions and their properties, Journal of Scientific and Engineering Research 5(9) (2018), 257-270.

[2] A. A. Atash, S. S. Barahmah and M. A. Kulib, On a new extensions of extended Gamma and Beta functions, International Journal of Statistics and Applied Mathematics 3 (2018), 14-18.

[3] M. A. Chaudhry and S. M. Zubair, Generalized incomplete gamma functions with applications, J. Comput. Appl. Math. 55 (1994), 99-124.

[4] M. A. Chaudhry, A. Qadir, M. Rafique and S. M. Zubair, Extension of Euler's beta function, J. Comput. Appl. Math. 78 (1997), 19-32. 
[5] J. Choi, A. K. Rathie and R. K. Parmar, Extension of extended beta, hypergeometric and confluent hypergeometric functions, Honam Math. J. 36(2) (2014), 357-385.

[6] G. M. Mittag-Leffler, Sur la nouvelle function $E_{\alpha}(x)$, C. R. Acad. Sci. Paris 137 (1903), 554-558.

[7] T. R. Prabhakar, A singular integral equation with a generalized Mittag Leffler function in the kernel, Yokohoma Math. J. 19 (1971), 7-15.

[8] P. I. Pucheta, A new extended beta function, International Journal of Mathematics and its Applications 5(3-C) (2017), 255-260.

[9] G. Rahman, G. Kanwal, K. S. Nisar and A. Ghaffar, A new extension of Beta and hypergeometric functions, 2018. doi:10.20944/preprints201801.0074.v1.

[10] M. Shadab, S. Jabee and J. Choi, An extension of Beta function and its application, Far East J. Math. Sci. 103(1) (2018), 235-251.

[11] H. M. Srivastava and H. L. Manocha, A Treatise on Generating Functions, New York: Halsted Press, 1984.

[12] A. Wiman, Über den Fundamentalsatz in der Teorie der Funktionen $E^{a}(x)$, Acta Math. 29(1) (1905), 191-201. 